\title{
ATIVIDADES DE LAZER EM ADULTOS QUILOMBOLAS DE UMA REGIÃO BAIANA
}

Recebido em: 06/02/2021

Aprovado em: 28/05/2021

Licença:@() (1)

Késsia Mirian Jesus de Oliveira
Leila Maria Prates Teixeira Mussi
Claudio Bispo de Almeida
${ }^{3}$
Ricardo Franklin de Freitas Mussi ${ }^{4}$
Universidade do Estado da Bahia (UNEB) - Campus Guanambi
Guanambi - BA - Brasil

RESUMO: O presente estudo objetivou analisar as atividades de lazer realizadas por quilombolas baianos da microrregião de Guanambi, Bahia, Brasil. Foram utilizados dados de um estudo transversal, com amostra de 850 participantes com idade $\geq 18$ anos. Como variável dependente, adotou-se atividades de lazer. Os dados foram analisados por meio de estatística descritiva e teste de associação (Qui-quadrado ou Exato de Fisher). Em todas as análises foram consideradas como significância estatística o valor de $\mathrm{p}<0,05$. Os resultados mostraram que $78,0 \%$ dos participantes relataram praticar lazer regularmente. Quanto ao tipo de atividade de lazer, as mais praticadas foram as sociais $(51,0 \%)$, enquanto que, as menos citadas foram as culturais $(3,0 \%)$. As variáveis associadas com alguma prática de lazer foram: ser adulto $(\mathrm{p}=0,047)$; situação conjugal com companhia marital $(p=0,047)$; e saber ler ou escrever $(p=0,003)$. Conclui-se que a amostra avaliada desenvolve atividades de lazer em suas comunidades, as quais se associaram ao seu cotidiano e às relações sociais culturalmente estabelecidas.

PALAVRAS-CHAVE: Estudos Transversais. Grupo com Ancestrais do Continente Africano. Atividades de Lazer.

\section{LEISURE ACTIVITIES IN QUILOMBOLA ADULTS IN A BAHIA REGION}

ABSTRACT: This study aimed to analyze leisure activities carried out by quilombolas from Bahia in the micro-region of Guanambi, Bahia, Brazil. Data from a cross-sectional study were used, with a sample of 850 participants aged $\geq 18$ years. As a dependent variable, leisure activities were adopted. The data were analyzed using descriptive

\footnotetext{
${ }^{1}$ Licenciada em Educação Física pela Universidade do Estado da Bahia.

${ }^{2}$ Doutoranda em Memória pela Universidade Estadual do Sudoeste da Bahia. Mestra em História pela Universidade do Estado da Bahia. Docente na Faculdade Santo Agostinho.

${ }^{3}$ Doutorando em Enfermagem e Saúde pela Universidade Estadual do Sudoeste da Bahia. Mestre em Educação Física pela Universidade Federal de Santa Catarina. Docente na Universidade do Estado da Bahia.

${ }^{4}$ Docente no Programa de Pós-Graduação em Ensino, Linguagem e Sociedade da Universidade do Estado da Bahia. Doutor em Educação Física pela Universidade Federal de Santa Catarina. Líder do Grupo de Estudos, Pesquisa e Extensão em Educação, Cultura e Saúde (GEPEECS/CNPq).
} 
statistics and the association test (Chi-square or Fisher's Exact). In all analyzes, $p<0.05$ was considered as statistically significant. The results showed that $78.0 \%$ of the participants reported practicing leisure regularly. As for the type of leisure activity, the most practiced were social $(51.0 \%)$, while the least cited were cultural $(3.0 \%)$. The variables associated with some leisure practice were: being an adult $(\mathrm{p}=0.047)$; marital status with marital companionship $(\mathrm{p}=0.047)$; and how to read or write $(\mathrm{p}=0.003)$. It is concluded that the evaluated sample develops leisure activities in their communities, which are associated with their daily lives and culturally established social relationships.

KEYWORDS: Cross-sectional Studies. African Continental Ancestry Group. Leisure Activities.

\section{Introdução}

A discussão do lazer como dimensão da cultura, permite a compreensão de sua legitimidade nos mais diferentes segmentos populacionais. A cultura humana representa um campo de produção com várias perspectivas, com o lazer interagindo extensamente com a fruição dessas manifestações culturais (GOMES, 2003). Destarte, é necessário pensá-lo no contexto sociocultural, respeitando as características peculiares de cada sociedade, sem desconsiderar suas contradições e possibilidades.

O lazer constrói-se socialmente em um tempo/espaço, institui-se como expoente da cultura, compreendido como um fenômeno sócio-histórico-cultural, que através das transformações se estabelece na sociedade e em seus valores. O lazer é espaço/tempo de expressão, de realidade, de usufruto, de produção e de cultura (CARVALHO, 2005; MONTENEGRO; DIAS; PAIXÃO, 2017).

A Constituição Federal Brasileira (BRASIL, 1988) reconheceu o lazer como um direito, que está relacionado às questões sociais e culturais da população brasileira. Neste sentido, Gomes (2011) refere-se ao lazer como uma necessidade humana, que se aplica em uma esfera ampla de contribuições para as práticas sociais, portanto, integra a vida cotidiana das pessoas se agregando em diferentes culturas. De tal modo, o lazer proporciona "alternativas para enfrentar situações complexas como as desigualdades 
sociais, os conflitos armados, a marginalização, a destituição dos direitos sociais, a precarização do trabalho e as distintas formas de violência [...]” (GOMES, 2011, p.15).

Mesmo as atividades de lazer sendo uma prática social complexa constituída por diversos tipos de vivências culturais contextualizadas e historicamente situadas (GOMES 2014), percebe-se que algumas comunidades remanescentes de quilombos não conseguem usufruir deste direito. Em algumas comunidades quilombolas pode-se perceber a ausência de atividades diversificadas dessas práticas (SILVA; MENEZES, 2016). Em outras, encontram-se poucas atividades de lazer, muitas vezes resumidas ao futebol e outros jogos (NUNES; CHAVES, 2019).

O usufruto do lazer por comunidades negras, especificamente as que são reconhecidas como quilombolas, apesar do interesse crescente por sua discussão no campo acadêmico, ainda precisa avançar com enfoque nos costumes e manifestações. Destarte, estudo referente a atividades físicas de tempo livre, revelou que pessoas pouco ativas nessa dimensão tendem a comportamento sedentário, consequentemente a redução nas condições de saúde (MUSSI et al., 2015).

É relevante ressaltar que estas comunidades quilombolas são constituídas por grupos que desenvolveram, ao longo do tempo práticas culturais e modos de vida específicos em seus territórios, com identidade própria que definem as experiências vividas e o compartilhamento de suas trajetórias históricas comuns, o que possibilita a sua continuidade (MUSSI et al., 2015). Assim, os quilombos podem ser compreendidos como organizações comunitárias que se estabeleceram historicamente e/ou contemporaneamente em resposta a opressão da sociedade brasileira historicamente escravocrata, transformaram-se em espaço de resistência cultural e social.

Para Miranda (2018), por mais de dois séculos houve contestações na dinâmica política sobre o significado e conjunção dos quilombos no Brasil. Passando das 
interpretações de comunidades que agrupava negros foragidos e criminosos, para comunidades geograficamente isoladas, com moradias precárias e com produção exclusivamente agrícola, conquistando ressignificação, como comunidades símbolo de resistência e luta. O reconhecimento dos direitos (BRASIL, 1988) garantiu a regulamentação de sua identificação, reconhecimento, delimitação, e titulação de suas terras, assim como sua a reprodução cultural, social, religiosa, ancestral e econômica.

Contudo, o mito do embranquecimento e o racismo existente no Brasil, submetem as comunidades negras até os dias atuais, ao ranking de população negligenciada pelo poder público, no qual recebem pouco reconhecimento e enfrentam baixos indicadores socioeconômicos. O panorama social dessas comunidades, é de uma população que ainda luta pela igualdade de direitos, acesso a regularização de suas terras, da saúde e por uma aplicação de uma cidadania plena (FREITAS et al., 2011).

Diante das questões históricas que impõem uma condição de vulnerabilidade e diminuta disponibilidade de investigações/informações sobre as comunidades quilombolas, buscou-se, no presente estudo, um olhar voltado às práticas de lazer. Desta forma, emerge a indagação sobre quais práticas de atividades de lazer são desenvolvidas por adultos residentes em comunidades quilombolas de região baiana? Assim, tem-se como objetivo analisar as atividades de lazer realizadas por quilombolas baianos da microrregião de Guanambi, Bahia, Brasil.

\section{Métodos}

O presente estudo utiliza dados do estudo transversal de base populacional intitulado "Perfil Epidemiológico dos Quilombolas baianos", previamente autorizado pelo Comitê de Ética em Pesquisa com Seres Humanos da Universidade do Estado da 
Bahia, sob o parecer n. 1.386.019/2016, com coletas desenvolvidas de fevereiro a novembro de 2016.

O campo empírico de investigação a microrregião geográfica de Guanambi, Bahia, composta por 18 municípios, que contava com 42 quilombos contemporâneos certificados até o ano de 2016, distribuídos em 10 destes municípios. Diante da indisponibilidade de informações oficiais prévias relativas à quantidade de moradores dos quilombos desta microrregião baiana, a população foi estimada considerando 80 famílias por quilombo, com dois adultos (pessoas com idade >18 anos, de ambos os sexos) por família, sinalizando uma população de 6.720 adultos residentes nas comunidades quilombolas da região pesquisada.

O desenho amostral consistiu em duas etapas: sorteio dos quilombos (conglomerado) e, em seguida, coleta censitária. Inicialmente foi realizado sorteio aleatório dos quilombos. Por meio das respectivas associações de moradores, 14 unidades sorteadas permitiram visitações para a realização da pesquisa, quantitativo necessário para atender aos critérios amostrais estabelecidos.

Para o cálculo amostral foi adotada correção para população finita, prevalência de $50 \%$ para desfecho desconhecido, intervalo de confiança de 95\%, erro amostral de cinco pontos percentuais, efeito do 1,5 vez para conglomerado em um estágio, acréscimo de $30 \%$ para recusas e $20 \%$ para perdas e confundimento, determinando amostra mínima necessária de 818 participantes para garantir a representividade desta população.

Dentre os critérios de elegibilidade, teve-se que aqueles com deficiência cognitiva ou de comunicação independente, aparentes ou diagnosticadas, foram excluídos das entrevistas. As perdas foram definidas pela ausência resposta de alguma questão da entrevista. 
Assim, considerando todos os adultos nos quilombos elegíveis, as associações de moradores informaram a presença de 1.025 adultos residentes nas 14 comunidades sorteadas durante o período das coletas de dados. Todos foram convidados, sendo informados sobre os aspectos do estudo, garantindo igual probabilidade de participação. Compareceram voluntariamente às atividades e aceitaram participar por meio da assinatura ou fornecimento da impressão digital no Termo de Consentimento Livre e Esclarecido individual 850 quilombolas, compondo a amostra final. Não compareceram às atividades $17,07 \%$ dos convidados, caracterizando as recusas.

A coleta de dados foi desenvolvida por equipes compostas por profissionais e/ou estudantes universitários da área de saúde e ciências humanas conforme suas habilitações, após treinamento para sua respectiva função. As coletas foram realizadas em sistema de mutirão, durante dias estabelecidos pelas associações de moradores. A equipe de coleta de dados realizou entrevistas, calcadas em instrumento validado para população quilombola (BEZERRA et al. 2014). Mais descrições sobre a coleta de dados podem ser obtidas no manuscrito "Inquérito de saúde em população quilombola baiana: relato de uma experiência em pesquisa epidemiológica" (MUSSI et al., 2020).

Atividades de lazer (variável independente): a participação em atividades de lazer foi obtida pelas respostas a duas perguntas. A pergunta se "Você participa de atividades regulares de lazer?" (sim ou não) indicou a prática de alguma atividade de lazer. De maneira complementar, o questionamento sobre "Qual o tipo de atividade de lazer realizada?" permitia a indicação de atividades culturais (ir ao cinema, teatro, exposição e leitura de livros); atividades sociais (visita a amigos, festa, barzinho, jogos: baralho/dominó); atividades físicas (caminhadas, natação, prática de esportes, corrida, academia); e, atividades auto focadas (assistir TV ou ouvir rádio). 
Variáveis de análise: Variáveis sociodemográficas: sexo (feminino; masculino); grupo etário (<40 anos; >40 anos); situação conjugal (com companheiro; sem companheiro); escolaridade ( $>4$ anos de estudo; $<4$ anos de estudo); renda familiar $(\geq 01$ salário mínimo; $\leq 01$ salário mínimo), para salário mínimo de $\mathrm{R} \$ 880,00$ em vigor no Brasil no ano de 2016.

Os dados foram analisados, inicialmente, por meio de estatística descritiva. $\mathrm{Na}$ sequência, realizou-se o teste de associação (Qui-quadrado ou Exato de Fisher) entre a variável independente e as variáveis dependentes. Em todas as análises considerou-se como significância estatística o valor de $\mathrm{p}<0,05$.

\section{Resultados}

Dentre os 850 adultos quilombolas participantes, 820 responderam à pergunta relativa à participação de atividades de lazer. Neste sentido, 78,0\% declararam praticar lazer regularmente (TABELA 1).

Quanto ao tipo de atividade de lazer, as mais praticadas são as sociais $(51,0 \%)$, como por exemplo, visitar os amigos, festa, barzinho, jogos, dentre outras. Enquanto que as menos indicadas foram as culturais (leitura de livros, ir ao cinema, exposição, teatro, dentre outras) desenvolvidas por 3,0\% dos entrevistados (TABELA 1). 
Tabela 01: Distribuição de Frequências da Participação em Atividades Regulares de Lazer e Práticas Realizadas por Quilombolas da Microrregião de Guanambi, Bahia, Brasil, 2016. ( $\mathrm{N}=850)$.

\begin{tabular}{lc}
\hline \multicolumn{1}{c}{ Variáveis } & Lazer \% \\
\hline "Você participa de atividades de regulares de lazer?” & \\
Sim & $78,0 \%$ \\
Não & $22,0 \%$ \\
“Qual o tipo de atividade de lazer realizada?” & \\
Atividades culturais & $3,0 \%$ \\
Atividades sociais & $51,0 \%$ \\
Atividades físicas & $21,0 \%$ \\
Atividades auto focadas (assistir TV ouvir rádio) & $25,0 \%$ \\
\hline
\end{tabular}

Fonte: Dados da pesquisa.

O perfil sociodemográfico da população é predominantemente composto por adultos $79,3 \%$, do sexo feminino $78,0 \%$, com relacionamento conjugal $76,3 \%$, com renda familiar de até um salário mínimo $78,6 \%$, e alfabetizados $74,8 \%$.

As análises de associação indicaram relação estatisticamente significativa entre prática de atividade de lazer com faixa etária $(\mathrm{p}=0,047)$, situação conjugal $(\mathrm{p}=0,047)$ e ao fato de saber ler/escrever $(\mathrm{p}=0,003)$. Neste sentido, os quilombolas adultos, sem companhia marital e alfabetizados são mais propensos a praticarem alguma atividade de lazer (TABELA 2).

Tabela 2: Associação entre Prática de Atividade de Lazer e Variáveis Sociodemográficas de Quilombolas da Microrregião de Guanambi, Bahia, Brasil, 2016. $(\mathrm{N}=\mathbf{8 5 0})$.

\begin{tabular}{lcccc}
\hline Variáveis & $\begin{array}{c}\text { Sim } \\
\%(\mathrm{n})\end{array}$ & $\begin{array}{c}\text { Não } \\
\%(\mathrm{n})\end{array}$ & $\begin{array}{c}\text { Total } \\
\%(\mathrm{n})\end{array}$ & Valor de $\mathrm{p}$ \\
\hline Sexo & & & 0,973 \\
\hline Masculino & $78,0(248)$ & $22,0(70)$ & $100,0(318)$ & \\
\hline Feminino & $77,9(391)$ & $22,1(111)$ & $100,0(502)$ & \\
\hline Faixa etária & & & $100,0(671)$ & \\
\hline $18-59$ anos & $79,3(532)$ & $16,7(139)$ & $100,0(149)$ & \\
\hline$\geq 60$ anos & $71,8(107)$ & $28,2(42)$ & & 0,047 \\
\hline Situação Conjugal & & & $100,0(630)$ & \\
\hline Com companheiro & $76,3(481)$ & $23,7(149)$ & $100,0(190)$ & \\
\hline Sem companheiro & & $16,8(32)$ & & \\
\hline
\end{tabular}




\begin{tabular}{|c|c|c|c|c|}
\hline $\begin{array}{l}\text { Renda familiar mensal (em } \\
\text { Reais)* }\end{array}$ & & & & 0,692 \\
\hline Até R $\$ 880,00$ & $78,6(452)$ & $21,4(123)$ & $100,0(575)$ & \\
\hline$>\mathrm{R} \$ 880,00$ & $77,1(118)$ & $22,9(35)$ & $100,0(153)$ & \\
\hline Sabe ler/escrever & & & & 0,003 \\
\hline$\overline{\mathrm{Sim}}$ & $74,8(478)$ & $25,2(161)$ & $100,0(593)$ & \\
\hline Não & $63,5(115)$ & $36,5(66)$ & $100,0(227)$ & \\
\hline
\end{tabular}

Fonte: Dados da pesquisa.

*Valor do salário mínimo vigente no ano da pesquisa (2016).

\section{Discussão}

Entre os residentes das comunidades remanescentes de quilombos da região baiana participante do presente estudo, foi identificada maior prevalência de praticantes de atividades de lazer relacionada com as atividades sociais.

As atividades sociais identificadas como primeira opção geral de atividade para práticas de lazer $(51,0 \%)$ pelas comunidades da microrregião de Guanambi, Bahia, seguem as características e especificidades desse povo, os festejos religiosos, a musicalidade, as danças e os jogos esportivos, são algumas das atividades que povoam o cotidiano dessas comunidades.

A maior prevalência em atividades sociais, encontrada nessa pesquisa, corrobora com estudo do Estado do Paraná, o qual evidenciou que os modos de lazer de muitos adultos estão relacionados ao que a igreja e seus cultos oferecem (RANGEL; MIRANDA; LARA, 2014). Tais práticas de caráter religioso, são caracterizadas pela forte presença do sincretismo. Tais práticas sociais se referem a forma que uma sociedade se estrutura através de normas e costumes. Em um mesmo povo convivem classes sociais diferentes, e essas diferenças podem engendrar preconceitos, discriminação e até mesmo formas ideológicas diferentes (MUNANGA, 2010) e assim, 
formas de reprodução histórica e de relações sociais vão sendo moldadas conforme as necessidades se apresentam.

Neste contexto, o lazer integra a vida cotidiana das pessoas, representando a necessidade de fruir ludicamente, as incontáveis práticas culturais constituídas, socialmente em cada contexto. Essa necessidade pode ser satisfeita de múltiplas formas, segundo os valores e interesses dos sujeitos, grupos e instituições em cada contexto histórico, social e cultural (GOMES, 2011).

Assim, as tradições, as festas, as brincadeiras, o lazer e outros elementos culturais estão impregnados ou revestidos por valores, símbolos e signos em atividades cotidianas sociais (MASCARENHAS, 2003). Têm-se, como exemplo destas práticas de lazer as práticas religiosas (MENDES; CAVAS, 2018), as atividades sociais ligadas à obtenção de alimentos e recursos financeiros como a pesca e o plantio, podem ser associados ao lazer, relevando a relação direta do trabalho como o modo de vida dessas comunidades, inclusive em comunidades remanescentes de quilombos (FREITAS; SILVA; GALVÃO, 2009).

Nota-se que as práticas de lazer nem sempre passam em atividades coletivas, no presente estudo, a prevalência entre comportamento individual de assistir TV ou rádio se estabeleceu como a segunda atividade de lazer mais praticada. Pesquisa realizada por Farah et al. (2013), com trabalhadores industriários, mostrou que o tempo gasto excessivamente em frente à TV pode acentuar o isolamento social e ser predisposição para percepção de estresse.

Além disso, percebe-se que a TV aberta, na qual, veiculam-se comerciais e programação para determinado público, como por exemplo, a classe trabalhadora, enquanto que, nos canais por assinatura, o direcionamento de sua programação é para a classe com maior poder aquisitivo. Esse meio de comunicação pode ser fonte de 
informações, diversão/entretenimento e cultura, mas pode se tornar espaço de consumo de conteúdos culturais de forma passiva e alienante, manipulação de valores e modos de vida (FONSECA, 2019).

O presente estudo evidenciou que a prática atividade física se estabeleceu como a terceira atividade de lazer realizada nas comunidades. Resultado similar ao observado em pesquisa realizada no sudoeste baiano, onde se encontrou que as prevalências e os fatores associados à atividade física apresentaram baixo percentual de atividade física no lazer dos quilombolas estudados (BEZERRA et al., 2015).

Em estudo realizado na comunidade remanescente Tomé Nunes, na Bahia, as atividades físicas de tempo livre foram o objeto principal investigado. Os resultados indicaram que o futebol foi à forma de lazer mais desenvolvida entre os moradores, tendo participação igualitária tanto masculina, quanto feminina (MUSSI et al., 2015). Outros estudos também apontaram o futebol como prática presente nas comunidades quilombolas (FREITAS; SILVA; GALVÃO, 2009; LARA; PIMENTEL, 2013; RANGEL; MIRANDA; LARA, 2014; NUNES; CHAVES, 2019).

Sabe-se que o futebol além do seu viés esportivo/competitivo é uma manifestação cultural, que revela características de malicia e alegria, protagonizando contornos de um processo de identificação construído e engendrado por esses diferentes agentes sociais em interação (TOLEDO, 2000). Na comunidade Arturos pôde-se perceber forte relação com a modalidade esportiva, sendo o campo de futebol espaço que acontecem os campeonatos e as "peladas", assim como espaço de encontro com os amigos, tanto para jogadores, quanto para quem vai para torcer e assistir as partidas (NUNES; CHAVES, 2019).

Desta forma, na comunidade paraense de Santarém, o futebol é a prática de maior adesão dos homens (FREITAS; SILVA; GALVÃO, 2009) e em algumas 
comunidades paranaenses o futebol é jogado em campos improvisado pelos moradores (LARA; PIMENTEL, 2013), sendo esta uma característica comum em todas as comunidades, campos de chão batido, com traves improvisadas pelos próprios moradores. Percebe-se o futebol utilizado como uma forma de lazer presente nas comunidades remanescentes de quilombos, sendo praticado por crianças, jovens e adultos, tendo a participação feminina presente.

No presente estudo, outra atividade física que foi evidenciada nos momentos de lazer foi a caminhada. Uma pesquisa realizada no sudoeste baiano mostrou a caminhada como a segunda atividade mais praticada (MUSSI et al., 2015). As pesquisas mostram que a caminhada tem sido mais frequente entre as mulheres (DEL DUCA et al., 2014; MUSSI et al., 2015). Percebe-se que a facilidade em praticar esta atividade pode contribuir para uma maior adesão.

As atividades culturais foram às práticas de lazer menos citadas nas comunidades da microrregião de Guanambi. Traços fortes da cultura africana podem ser encontrados em variados aspectos da cultura brasileira, como por exemplo, a música popular, a religião, a culinária, o folclore e as festividades populares. Mesmo passando tradicionalmente por desvalorização os aspectos culturais de influência africana se estabelecem em nossa sociedade. Contudo, esses aportes culturais devem ser sempre resgatados positivamente, desconstruindo imagens negativas que fizeram delas (MUNANGA, 2012).

Percebe-se que em algumas comunidades, a exemplo de Arturos, situada em Contagem, Minas Gerais, Brasil, é realizada a capoeira na qual as crianças e os jovens participam, e toda a comunidade se reúne para participar da roda acompanhando a música com palmas (NUNES; CHAVES, 2019). A capoeira trata-se de uma das manifestações culturais da corporeidade humana, a qual é baseada em um diálogo 
corporal. Outrora, foi uma manifestação marginalizada e reprimida por toda a sociedade, perseguida e violentada pela polícia, contudo ela resistiu, obteve conquistas e reconhecimento pelo seu valor histórico de resistência, educação e cultura do povo negro (CAMPOS, 2009).

Na presente pesquisa, as variáveis associadas com algum tipo de prática de lazer foram: ser adulto, situação conjugal com companhia marital e saber ler ou escrever. O lazer objeto de estudo dessa pesquisa, historicamente tem sido entendido e tratado, pelo estado como pela sociedade, como direito de menor valor (ATHAYDE; MASCARENHAS; SALVADOR, 2015), mesmo estando entre os direitos sociais desde 1988 (BRASIL, 1988), se este é negligenciado para a população caucasiana imagina para população negra.

Assim, o presente estudo mostrou maior prevalência de quilombolas praticantes de atividade de lazer, quando comparadas com outras investigações com populações similares realizadas por Mussi et al. (2015) no alto sertão baiano e por Bezerra et al. (2015) no sudoeste da Bahia, nas quais foram verificadas menor frequência (47,3\% e 13,1\%, respectivamente). Assim, é possível supor que essas diferenças podem ser decorrentes, em grande parte, pela maior abrangência da população alvo nos levantamentos realizados neste estudo, assim como dimensionando a análise para os diversos fatores do lazer.

A prevalência de atividades de lazer revela-se mais elevada entre os adultos, esse achado corrobora com os resultados dos estudos na área do lazer (NUNES; CHAVES, 2019; BEZERRA et al. 2015; MUSSI et al. 2015; FARAH et al. 2013). Pode-se especular que, na faixa etária menor (18 a 59 anos), esta diferença de prática de lazer decorra do maior envolvimento dos mais jovens em afazeres sociais, culturais, de 
deslocamento, entre outras. Assim, adultos mais velhos apresentam maior exposição a comportamentos sedentários, decorrente aos acometimentos causados pela idade.

Entretanto, podem existir ações sociais que promovam programas de convívio e fortalecimento de vínculos que reúnem idosos em encontros e oficinas para o desenvolvimento de atividades de lazer. Na comunidade quilombola de Arturos, é visualizada a existência desse grupo, no qual mulheres mais velhas se reúnem para conversar, distrair, realizando costura e bordados, se tornando momento de relação social e desenvolvimento de atividades de lazer (NUNES; CHAVES, 2019).

Para a variável estado conjugal, no presente estudo, os solteiros foram mais ativos no lazer, resultado semelhante ao das comunidades do sudoeste da Bahia no domínio de atividade física (BEZERRA et al., 2015). É provável que os solteiros, por não se prenderem nas obrigações familiares, predispõem de maior tempo livre para as práticas de lazer. Entretanto, cabe ressaltar que essas vivências também representam um recorte socioeconômico e cultural, pois para determinados sujeitos e coletivos, os papeis sociais podem ser outros. Assim como pode ser justificado a probabilidade que indivíduos casados da comunidade da microrregião de Guanambi, por trabalharem com agricultura de subsistência permaneçam maior período no trabalho e em casa e não considerem realizar essas atividades.

O presente estudo mostrou que quanto maior o nível escolar, maior a probabilidade dos participantes praticarem atividades de lazer. De acordo Pitanga et al. (2014), pessoas com mais anos escolarização tem maior acesso a conhecimentos e a melhores condições materiais de vida, o que favorece a adoção de prática e hábitos saudáveis. Quando se tem acesso ao capital cultural este pode estar vinculado ao nível de escolaridade, a tendência é que a concepção e a importância ao lazer sejam diferenciadas (BRAGA; SANTOS, 2019). 
A pesquisa não apresentou associação entre sexos, não expondo resultado significante, no entanto, estudos mostram maior participação masculina nas atividades de tempo livre e as mulheres no domínio doméstico (PITANGA et al., 2014; DEL DUCA et al., 2014). A problemática existente da desigualdade de gênero afeta diretamente o acesso ao lazer. Na população em geral as mulheres dispõem de menor tempo do que os homens, o que fragmenta as oportunidades de se envolverem em atividades recreativas (BARBOSA; LIECHTY; PEDERCINI, 2013), o trabalho doméstico ou a dupla jornada de trabalho no qual as mulheres são submetidas acaba por reduzir o seu tempo de lazer.

\section{Considerações Finais}

Conclui-se que, as atividades de lazer mais praticadas pelos quilombolas avaliados foram as sociais, enquanto as menos indicadas foram as culturais. Houve associação das variáveis: faixa etária, constatando que ser adulto (18 a 59 anos) aumenta a probabilidade de praticar atividades de lazer; ser solteiro, como aqueles que tendem a participar mais de atividades de lazer; e, possuir maior escolarização, aumenta a possibilidade da prática de lazer. Assim, percebe-se que a amostra avaliada desenvolve atividades de lazer em suas comunidades, as quais se associaram ao seu cotidiano e às relações sociais culturalmente estabelecidas.

O presente estudo se torna relevante para o conhecimento e entendimento da predominância de atividades de lazer nas comunidades negras. Dessa forma, os resultados obtidos podem servir de base para novos estudos, visto o baixo número de publicações relacionado ao tema. Os dados da presente pesquisa podem ser utilizados por líderes comunitários das comunidades quilombolas e gestores públicos como forma 
de embasamento de programas e ações de intervenção em prol do desenvolvimento de atividades de lazer nas comunidades remanescentes de quilombos pesquisadas.

\section{REFERÊNCIAS}

ATHAYDE, P.; MASCARENHAS, F.; SALVADOR, E. Primeiras aproximações de uma análise do financiamento da política nacional de esporte e lazer no Governo Lula. Revista Brasileira de Ciências do Esporte, v. 37, n.1, p.2-10, 2015.

BARBOSA, C.; LIECHTY, T.; PEDERCINI, R. Restrições ao Lazer Feminino: Particularidades das Experiências de Lazer de Mulheres Homossexuais. LICERE, v. 16, n. 2, p. 2013.

BEZERRA, V. M. et al. Domínios de atividade física em comunidades quilombolas do sudoeste da Bahia, Brasil: estudo de base populacional. Caderno de Saúde Pública, Rio de Janeiro, v.31, n.6, p. 1213-1224, 2015.

et al. Inquérito de Saúde em Comunidades Quilombolas de Vitória da Conquista, Bahia, Brasil (Projeto COMQUISTA): aspectos metodológicos e análise descritiva. Ciência e saúde coletiva, v.19, n.6, p.1835-1847, 2014.

BRAGA, I. F.; SANTOS, A. R. B. dos. Concepções de Lazer sob a Perspectiva dos Adultos. Licere, v. 22, n. 4, p. 285-316, 2019. DOI: http://doi.org/10.35699/19813171.2019.16270.

BRASIL. Constituição (1988). Constituição da República Federativa do Brasil, 1988. Brasília: Senado Federal, Centro Gráfico, 1988. 292p.

CAMPOS, Hellio. Capoeira Regional: a escola de Mestre Bimba. Salvador: EDUFBA, 2009.

CARVALHO, Yara M. Lazer e saúde. Brasília: SESI/DN, 2005.

DEL DUCA, G. F. et al. Atividades físicas no lazer entre adultos de Florianópolis, Santa Catarina, Brasil: estudo populacional sobre as características das práticas e de seus praticantes. Ciência e saúde coletiva, v. 19, n. 11, p. 4595-4604, 2014.

FARAH, B. Q. et al. Percepção de estresse: associação com a prática de atividades físicas no lazer e comportamentos sedentários em trabalhadores da indústria. Revista Brasileira de Educação Física e Esporte, v.27, n.2, p.225-34, 2013.

FONSECA, J. L. A Televisão na Perspectiva dos Estudos do Lazer: um levantamento dos artigos publicados nas revistas Licere e RBEL. Licere, v.22, n.3, 2019. DOI: http://doi.org/10.35699/1981-3171.2019.15315.

FREITAS, D. B.; SILVA, J. de M.; GALVÃO, E. F. C. A relação do lazer com saúde nas comunidades quilombolas de Santarém. Revista Brasileira de Ciência do Esporte, v. 30, n. 2, p. 89-105, 2009. 
FREITAS, D. A; et al. Saúde e comunidades quilombolas: uma revisão de literatura. Revista CEFAC, v. 13, n. 5, p. 937-943, 2011.

GOMES, C. L.; MELO, A. de. Lazer no Brasil: trajetória de estudos, possibilidades de pesquisa. Movimento, v. 9, n. 1, p.23-44, 2003.

GOMES, C. L. Estudos do Lazer e Geopolítica do Conhecimento. Licere, v.14, n.3, p.1-25, 2011. DOI: http://doi.org/10.35699/1981-3171.2011.762.

Lazer: necessidade humana e dimensão da cultura. Revista Brasileira de Estudos do Lazer, v. 1, n. 1, p. 3-20, 2014.

LARA, M. L.; PIMENTEl, G. G. de A. Políticas Públicas de Esporte e Lazer: em comunidades quilombolas no Paraná. Maringá: Eduem, 2013.

NUNES, R. R.; CHAVES, E. Lazer e cultura: o cotidiano da comunidade dos Arturos. Licere, v.22, n.1, p.231-262, 2019. DOI: http://doi.org/10.35699/19813171.2019.12321.

MASCARENHAS, F. O pedaço sitiado: cidade, cultura e lazer em tempos de globalização. Revista Brasileira de Ciência do Esporte, v. 24, n. 3, p. 121-143, 2003.

MENDES, D. S.; CAVAS, C. S. T. Benzedeiras e benzedeiros quilombolasconstruindo identidades culturais. Interações, v. 19, n.1, p. 3-14, 2018.

MIRANDA, S. A. de. Quilombos e Educação: identidades em disputa. Educar em Revista, v. 34, n. 69, p. 193-207, 2018.

MONTENEGRO, G. M.; DIAS, M. C.; PAIXÃO, H. T. da. Entre Rio, Corpo e Lazer: O Futlama em Questão. Licere, v.20, n 4, p.238-260, 2017. DOI: http://doi.org/10.35699/1981-3171.2017.1733.

MUNANGA, K. Negritude e identidade negra ou afrodescendente: um racismo ou avesso? Revista da ABPN, v.4, n.8, p. 06-14, 2012.

Teoria social e relações raciais no Brasil contemporâneo. Cadernos Penesb, n.12, p.169-203, 2010.

MUSSI, R. F. de F. et al. Atividades Físicas Praticadas no tempo livre em comunidade quilombolas do alto sertão baiano. Licere, Belo Horizonte, v.18, n.1, p. 157-187

2015.DOI: http://doi.org/10.35699/1981-3171.2015.1080.

MUSSI, R. F. F. et al. Inquérito de saúde em população quilombola baiana: relato de uma experiência em pesquisa epidemiológica. Saúde e Pesquisa, v.13, n.3, p.675-685, 2020.

PITANGA, F. G. et al. Prevalência e fatores sociodemográficos e ambientais associados à atividade física no tempo livre e no deslocamento adultos. Motricidade, v. 10, n.1, p. 3-13, 2014. 
RANGEL, R.; MIRANDA, A. C. M. de; LARA, L. M. Política Pública de Esporte e Lazer no Feixo: Experiências de Pesquisa em uma Comunidade Quilombola no Paraná. Licere, v. 17, n. 1, p.1-31, 2014. DOI: http://doi.org/10.35699/19813171.2014.627.

SILVA, R.A; MENEZES, J.A. Os significados do uso de álcool entre jovens quilombolas. Revista Latinoamericana de Ciencias Sociales, Niñez y Juventud, v.14, n.1, p.493-504, 2016.

TOLEDO, L. H. de. No país do futebol. Rio de Janeiro: Jorge Zahar, 2000.

\section{Endereço dos (as) Autores (as):}

Késsia Mirian Jesus de Oliveira

Endereço Eletrônico: kessiamirian@outlook.com

Leila Maria Prates Teixeira Mussi

Endereço Eletrônico: lmprates@ @otmail.com

Claudio Bispo de Almeida

Endereço Eletrônico: claudio_bispo_de_almeida@hotmail.com

Ricardo Franklin de Freitas Mussi

Endereço Eletrônico: rimussi@yahoo.com.br 